DOI 10.31489/2021No1/16-28

UDC 539.199, 544.723.2

\title{
REARRANGEMENT OF THE CONFORMATIONAL STRUCTURE OF POLYAMPHOLYTES ON THE SURFACE OF A METAL NANOWIRE IN A TRANSVERSE MICROWAVE ELECTRIC FIELD
}

\author{
Kruchinin N. Yu., Kucherenko M.G. \\ Center of Laser and Informational Biophysics, Orenburg State University, Orenburg, 460018 Russia, \\ kruchinin_56@mail.ru, clibph@yandex.ru
}

\begin{abstract}
Molecular dynamics has been employed to study the rearrangement of the conformational structure of polyampholytes adsorbed on the surface of a gold nanowire with a periodic change in time of its polarity in the transverse direction at an ultrahigh frequency. The radial distributions of the atomic density of the polypeptide and its angular distributions on the nanowire surface have been calculated. At high temperatures, temporary fluctuations in the conformational structure of the adsorbed polyampholyte polypeptide were observed. In this case, for half the period of the nanowire polarity change, the macrochain conformation changed from dense enveloping of the nanowire to an elongated conformational structure along the dipole moment of the nanowire. At low temperature and the nanowire dipole moment, the swelling of the fringe of the adsorbed polyampholyte was observed with a displacement of most of its links to one side with respect to the plane perpendicular to the direction of the nanowire dipole moment and passing through its axis. At low temperature and high values of the nanowire dipole moment, the polyampholyte polypeptide was desorbed from the nanowire surface. An analytical model of conformational rearrangements of a polyampholyte Gaussian chain in the form of an external field perturbation theory is presented.
\end{abstract}

Keywords: polyampholytes, metal nanowire, conformational changes, molecular dynamics, microwave electric field

\section{Introduction}

Hybrid nanosystems consisting of plasmonicnanoobjects, in particular, gold nanorods and nanowires with adsorbed macromolecular chains, are widely used as elements of nanoelectronic devices, as well as in the creation of various chemical sensors [1-7]. At present, considerable efforts have been focused on increasing the sensitivity and expanding the possibilities of detecting sensors based on the effect of surface plasmon resonance and surface-enhanced Raman scattering based on such hybrid nanosystems. In this case, both photoactive molecules and plasmonic nanoparticles must be bound to the macrochain [8-13]. Of particular interest is the control of changes in the conformational structure of adsorbed polyampholytes on a conducting surface both by a static electric field and by electromagnetic radiation.

In an electric field, the conformational structure of a polyampholyticmacrochain adsorbed on a solid surface will change under its influence [14-18]. When transverse electric field is imposed on a cylindrical metal nanowire, electric charges are induced on its surface, with their surface density being distributed in proportion to the cosine of the angle between the directions of the electric field vector and the normal to the nanowire surface. The arising field of the induced polarization strongly distorts the primary electric field near the nanowire. Such an uneven distribution of induced charges on the surface of a nanowire will have a significant effect on the conformational structure of polyampholyte macrochains adsorbed on its surface.

In the case of exposure to electromagnetic radiation on a nanowire, in which the electric field vector that is variable along the length remains perpendicular to its axis, the conformational structure of the polyampholyte macrochain will change in accordance with fluctuations in the density of charges induced by this field on the nanowire surface. Such a rearrangement of the conformational structure of the macromolecule near the nanowire becomes possible upon excitation of field oscillations in it in the microwave range in the direction transverse to the axis. At certain frequencies of electromagnetic radiation, a resonance of changes in the conformational structure of a polyampholyte macromolecule on the surface of a metal nanowire may appear [18]. 
In this work, circular-section gold nanowires are considered as metal adsorbents for polypeptides in connection with the specific properties of conducting nanocylinders as promising plasmonicnanoantennas. Similar objects with axial geometry are carbon nanotubes, the plasmon properties of which were considered by the authors in [19-20], where it was shown that such nanotubes perform the functions of a kind of electromagnetic waveguides. One-dimensional plasmon-polaritons can propagate along the surface of a conducting cylinder parallel to its axis, while only localized surface plasmons with well-studied properties can form in nanoparticles of a finite volume. Spherical metal nanoparticles with adsorbed polypeptide macrochains were considered by us earlier in [14, 16-18].

Therefore, such hybrid nanosystems, in which the conformational structure of adsorbed polyampholytes on the surface of a gold nanowire or nanorod can be rearranged under the influence of both a static electric field and a variable with an ultrahigh frequency, can find application in the creation of new and modification of existing sensors based on the effect of surface-enhanced Raman scattering or surface plasmon resonance, as well as luminescent optical units for measuring the concentration of molecular oxygen [21-22].

The goal of this work is to study the rearrangement of the conformational structure of polyampholyte polypeptides on the surface of a gold nanowire under the influence of electromagnetic radiation, in which the electric field vector is directed in the transverse direction with respect to the nanowire axis.

\section{Molecular dynamic simulation}

Molecular dynamics (MD) simulation of polyampholytic polypeptides on the surface of a gold nanowire was performed using the NAMD 2.14 software package [23]. The model of a gold nanowire was obtained by cutting out a cylinder with a radius of $1.5 \mathrm{~nm}$ and a length of $15.5 \mathrm{~nm}$ from a gold crystal, while the atoms of the nanowire remained fixed during the MD simulation.

Four polyampholytic polypeptides with different orders of charged amino acid residues were examined:

1) A400R100D100 polypeptide consisting of 400 Ala units (A), 100 Arg units (R, charge of $+1 e$ ), and 100 Asp units $(\mathrm{D}$, charge of $-1 e)$, with the two latter being uniformly distributed over the chain, with the total number of amino acid residues being equal to $600-\left(\mathrm{ADA}_{2} \mathrm{RA}\right)_{100}$;

2) A480R60D60 polypeptide consisting of 480 Ala units, 60 Arg units, and 60 Asp units, with the two latter being uniformly distributed over the chain, with the total number of amino acid residues being equal to $600-\left(\mathrm{A}_{2} \mathrm{DA}_{4} \mathrm{RA}_{2}\right)_{60}$;

3) A784R98D98 polypeptide consisting of 784 Ala units, 49 pairs of Arg units, and 49 pairs of Asp units, with the two latter being uniformly distributed over the chain, with the total number of amino acid residues being equal to $980-\left(\mathrm{A}_{4} \mathrm{R}_{2} \mathrm{~A}_{8} \mathrm{D}_{2} \mathrm{~A}_{4}\right)_{49}$;

4) A880R54D54 polypeptide consisting of 880 Ala units, 27 pairs of Arg units, and 27 pairs of Asp units, with the two latter being uniformly distributed over the chain, with the total number of amino acid residues being equal to $988-\mathrm{A}_{8}\left(\mathrm{~A}_{8} \mathrm{D}_{2} \mathrm{~A}_{16} \mathrm{R}_{2} \mathrm{~A}_{8}\right)_{27} \mathrm{~A}_{8}$.

The CHARMM22 force field [24] was used for the polypeptides. Noncovalent interactions with the gold nanoparticle were described by the Lennard-Jones potential parameterized in [25]. The van der Waals potential was cut off at a distance of $1.2 \mathrm{~nm}$ using the smoothing function between 1.0 and $1.2 \mathrm{~nm}$. Electrostatic interactions were calculated directly at a distance of $1.2 \mathrm{~nm}$; for larger distances, the particle mesh Ewald (PME) method [26] with a mesh step of $0.11 \mathrm{~nm}$ was employed. The entire nanosystemwas placed in a cube with edges of $24 \mathrm{~nm}$, filled with TIP3P water molecules [27].

At the initial moment of time, the polypeptide macromolecule was located in the form of a nonequilibrium coil near the surface of an unpolarized cylindrical gold nanowire. Three different starting coils were considered for each polypeptide. The MD simulation has yielded three equilibrium conformational structures for each considered polypeptide. The formation of equilibrium conformations was controlled by monitoring variations in the root-mean-square distance (RMSD) between atoms of a polypeptide in different conformations. Subsequently, these structures were used as starting configurations for MD simulation on the surface of a transversely polarized nanowire.

In the case of uniform polarization of the nanowire in the transverse direction, the local electric field was set through the change in the atomic charges on the surface of the cylindrical nanowire according to the cosine law in the cross section. The following induced dipole moment of the nanowire per unit length were obtained: $p_{1 \max }^{n w}=1.5 \mathrm{kD} / \mathrm{nm}, p_{2 \max }^{n w}=3.0 \mathrm{kD} / \mathrm{nm}$ и $p_{3 \max }^{n w}=6.0 \mathrm{kD} / \mathrm{nm}$. In this case, in the positively charged region of the polarized nanowire, the atoms acquired the maximum electric field-induced partial charges equal $+0.25 e,+0.5 e$ and, $+1.0 e$, accordingly. At the first stage, MD simulation was carried out 
with transverse polarization of the nanowire in a static electric field for all the considered polypeptides until an equilibrium conformational structure was obtained.

At the second stage, during the MD simulation, the densities of charges on the nanowire surface were periodically varied with time according to the sine law with oscillation period $\mathrm{T}=2.4 \mathrm{~ns}$ (oscillation frequency of $416.7 \mathrm{MHz}$ ) for 3 oscillation periods. Each oscillation period was divided into 8 equal time periods of $0.3 \mathrm{~ns}$, during which the field remained unchanged, and the dipole moment of the nanowire in a selected period was set by averaging it over the entire period duration. The dipole moment of the nanowire changed in the following sequence, starting from the starting conformation of the polypeptide obtained on an unpolarized nanowire: $+0.69 p_{\max }^{n w}$ (average value in the oscillation region from $\pi / 8$ to $3 \pi / 8$ ), $+0.97 p_{\max }^{n w}$ (from $3 \pi / 8$ to $5 \pi / 8$ ), $+0.69 p_{\max }^{n w}$ (from $5 \pi / 8$ to $7 \pi / 8$ ), 0 (from $7 \pi / 8$ to $9 \pi / 8$ ), $-0.69 p_{\max }^{n w}$ (from $9 \pi / 8$ to $11 \pi / 8$ ), $-0.97 p_{\max }^{n w}$ (from $11 \pi / 8$ to $13 \pi / 8$ ), $-0.69 p_{\text {max }}^{n w}$ (from $13 \pi / 8$ to $15 \pi / 8$ ), and 0 (from $15 \pi / 8$ to $17 \pi / 8$ ) [18]. The MD simulation was carried out at constant temperatures of 300 and $900 \mathrm{~K}$ (Berendsen thermostat) for each considered polypeptide.

Based on the obtained results of MD simulation, the radial dependences of the density of atoms of polypeptides were calculated, as well as the angular distributions of atoms of polypeptides with a step of 10 degrees over the cross section.

\section{Results of the study}

According to the results of MD simulation of polypeptides on the surface of an unpolarized gold nanowire, it was found that the polypeptides partially envelop the nanowire (Fig. 1a). The predominance of the location of the amino acid residues of the polypeptide on one side or the other of the nanowire depended on the starting location of the non-equilibrium macrochain coil, while the adsorption of polypeptide residues occurred regardless of their type. The radial distributions of the average density of polypeptide atoms (Fig. 2a) had a characteristic shape with a peak of the radial density distribution near the nanowire surface. The average angular distributions of polypeptide atoms over the cross section according to all the results of the MD simulation indicated a generally uniform distribution of atoms around the nanowire.

On the surface of a transversely polarized gold nanowire in a static electric field, such a rearrangement of the conformational structure of the adsorbed polyampholyte macrochain took place, in which, as the value of the induced dipole moment of the nanowire per unit of its length increased, more and more Asp amino acid residues were displaced toward the surface in the positively charged region of the nanowire (at the top in Figs. $1 \mathrm{~b}$ and 1c), and Arg amino acid residues were displaced to the surface in the negatively charged region of the nanowire (below in Figs. $1 \mathrm{~b}$ and 1c). In this case, the stretching of the macromolecular fringe along the dipole moment in the transverse direction to the nanowire was observed due to the repulsion of the residues of the same name with respect to the surface in the polar regions of the nanowire (Figs. 1b and 1c). This is most pronounced in polypeptides with a large distance between opposite charged amino acid residues in the macrochain.

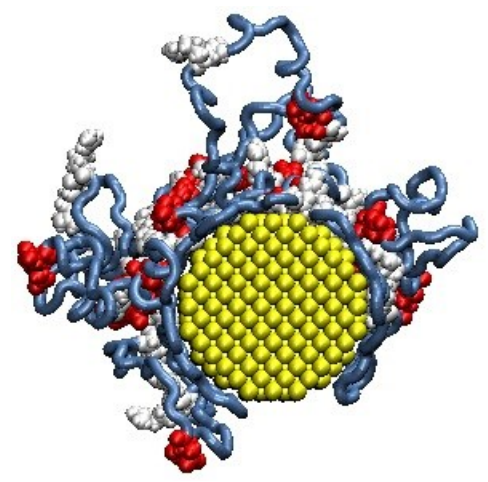

a

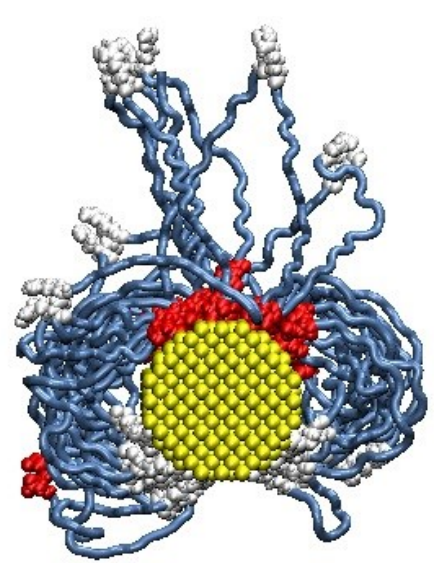

b

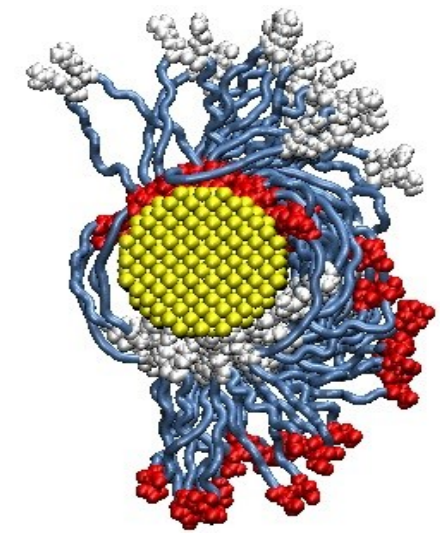

c

Fig.1. Polypeptide A880R54D54 on the surface of an unpolarized gold nanowire (a), polypeptides A880R54D54 (b) and A784R98D98 (c) on the surface of a polarized nanowire in a static electric field in the transverse direction (the dipole moment of the nanowire is directed upwards) after MD simulation (end view; the blue tube and white and red symbols denote Ala, Arg, and Asp residues, respectively). 
The radial distributions of the average density of polypeptide atoms on the surface of a polarized nanowire also differ from the case of adsorption on an unpolarized nanowire. Figure $2 b$ shows that the peak value of the radial distribution over all polypeptide atoms decreased by more than 5 times, and the profile of this distribution broadened significantly. This is due to the fact that the peaks of the radial distribution of the average density of the charged Arg and Asp amino acid residues of the polypeptide are located at the surface of the nanowire, and the profile of the radial distribution of the average density of Ala units is shifted from the surface. Figure $2 \mathrm{c}$ shows the average angular distributions of atoms of charged units of a polypeptide on the surface of a gold nanowire polarized in the transverse direction. The upper pole in Figure 1 along the cross-section of the nanowire corresponds to an angle of 0 degrees, and the lower pole corresponds to an angle of 180 degrees. It can be seen that a redistribution of Arg amino acid residues to the negatively charged region of the nanowire occurred on the polarized nanowire, and Asp units shifted to the positively charged region of the nanowire. The ratio of the thickness of the macromolecular fringe along the dipole moment of the nanowire to the thickness of the macromolecular layer in the orthogonal transverse direction was 1.4, 1.6, 1.9, and 2.2 times, respectively, for the A400R100D100, A480R60D60, A784R98D98, and A880R54D54 polypeptides. That is, the greater the distance between opposite charged links in the macrochain, the greater this ratio.

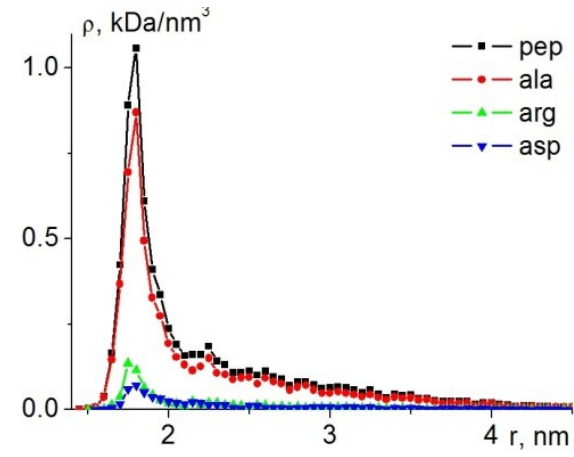

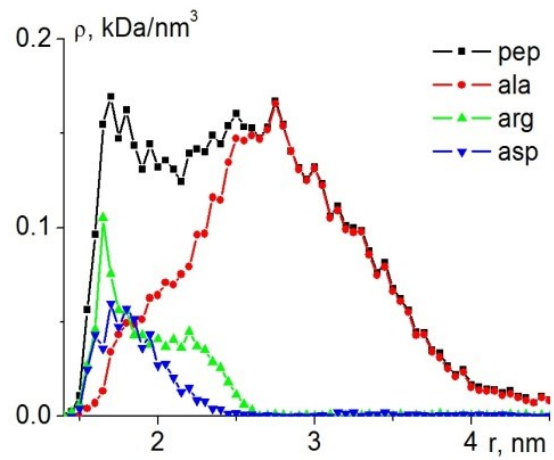

$\mathrm{b}$

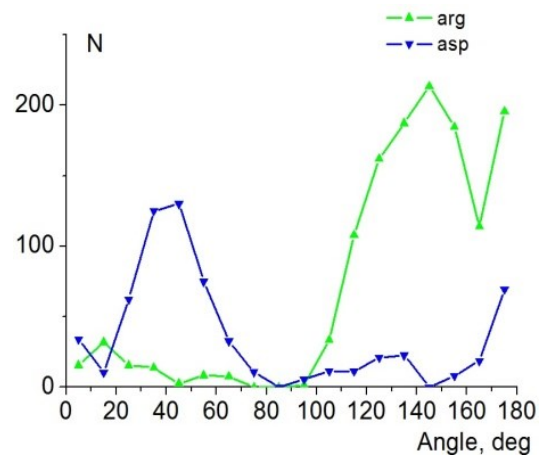

$\mathrm{c}$

Fig.2. Radial dependences of the average atomic density for A880R54D54 polypeptide on the surfaces unpolarized (a) and polarized in a static electric field in the transverse direction (b) gold nanowires with differentiation by the types of units. Dependences of the average angular distributions of atoms of charged units of the A880R54D54 polypeptide on

a polarized gold nanowire (c). In the figure: pep - dependence for all atoms of the polypeptide;ala, arg and asp dependences by types of amino acid residues.

According to the results of MD simulation with a temperature of $900 \mathrm{~K}$ with periodic changes in the polarization of the nanowire in the transverse direction, oscillations of the conformational structure of polyampholyte adsorbed on the surface were observed, similar to the temporal oscillations of conformations on the surface of a metal nanoparticle [18]. Such oscillations began to be clearly observed at the peak values of the induced dipole moment of the nanowire per unit of its length $p_{2 \max }^{n w}$ and higher for all the considered polypeptides, and at lower values of the dipole moment they were less pronounced. Figure 3 shows the conformational structures of the A784R98D98 polypeptide on the surface of the nanowire at different times according to the results of MD simulation with a peak dipole moment $p_{2 \max }^{n w}$.

It can be seen that for different directions of the dipole moment (Figs. 3a and 3b), the conformational structure has a mirror-like appearance with respect to the horizontal plane passing through the nanowire axis. It can also be seen that these conformational structures have a similar appearance to the conformational structure obtained as a result of MD simulation in a static field (Fig. 1c) with stretching the loops along the direction of the dipole moment. Figure $3 \mathrm{c}$ shows the final conformational structure of the A784R98D98 polypeptide, obtained as a result of MD simulation at the last time interval, when the nanowire dipole moment was zero. In this case, the polypeptide completely envelops the gold nanowire and has a similar appearance to the starting conformations on the surface of an unpolarized nanowire (Fig. 1a).

The radial distributions of the atomic density of the A784R98D98 polypeptide with differentiation by the types of units, averaged over all time intervals in the cases when the nanowire is not polarized (Fig.4a), polarized with a dipole moment $+0.97 p_{2 \max }^{n w}$ (Fig.4b), and $-0.97 p_{2 \max }^{n w}$ (Fig.4c), also have a similar form 
with radial distributions obtained on an unpolarized nanowire (Fig. 2a) and with polarization in a static electric field (Fig. 2b), respectively. In this case, the radial distributions of the average density of polypeptide atoms for opposite directions of the transverse polarization of the nanowire are similar to each other (Figs. $4 \mathrm{~b}$ and 4c). On the curves for the radial density distributions over all atoms of the A784R98D98 polypeptide show the vertical segments of the standard deviations. It can be seen that at the same phase of the change in the dipole moment of the nanowire, the deviations from the average value of the density of polypeptide atoms are small.

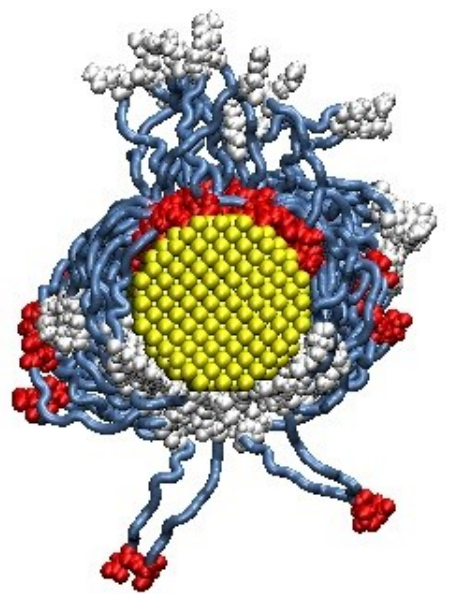

a

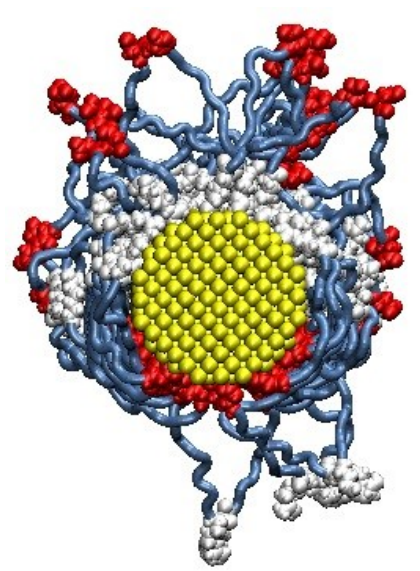

b

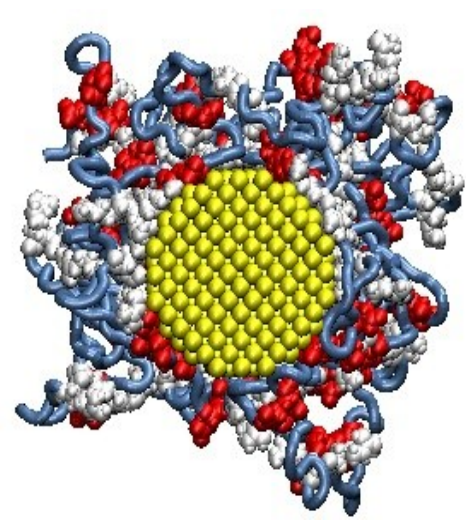

$\mathrm{c}$

Fig.3. Conformations of the A784R98D98 polypeptide during MD simulation with a periodic change in the nanowire polarization in the transverse direction at a temperature of $900 \mathrm{~K}$ : a) after $5.4 \mathrm{~ns}$, when the nanowire dipole moment is directed upward; b) after $6.6 \mathrm{~ns}$, when the dipole moment of the nanowire is directed downward; c) after $7.2 \mathrm{~ns}$, when the nanowire is not polarized.
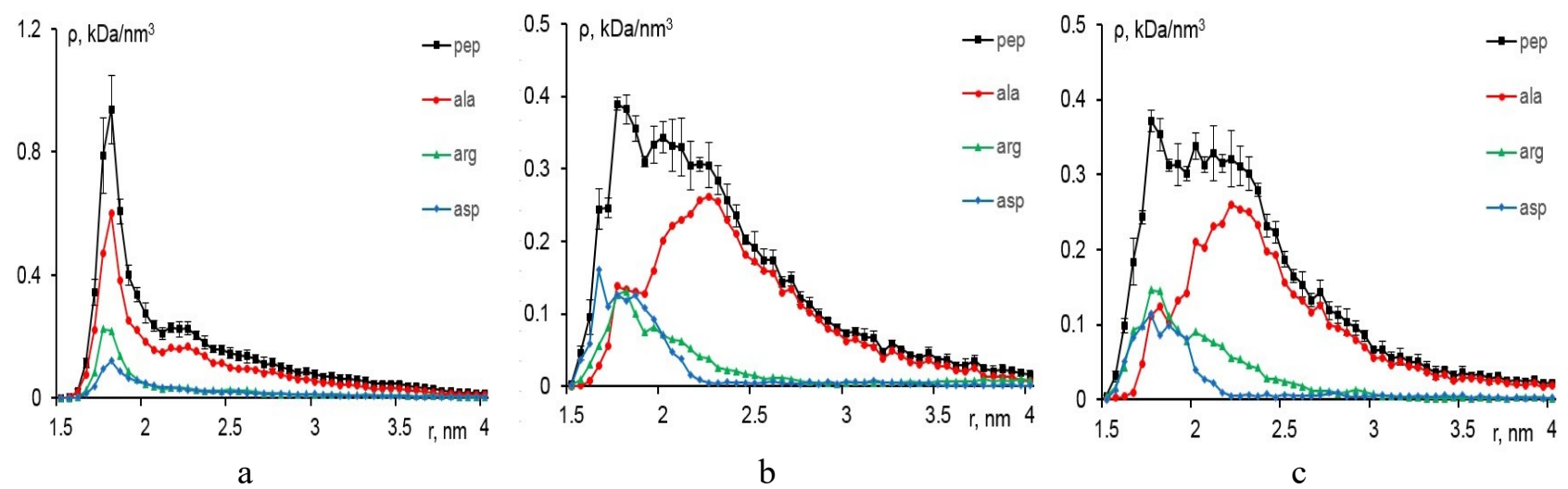

Fig.4. Radial distributions of the average density of atoms of the A784R98D98 polypeptide on the surface of a gold nanowire at the end of the MD simulation at $900 \mathrm{~K}$ on time intervals in cases where the nanowire is not polarized (a),

is polarized in the transverse direction with a dipole moment $+0.97 p_{2 \max }^{n w}$ (b) and $-0.97 p_{2 \max }^{n w}$ (c). In the figure:

vertical segments - an estimate of the standard deviation.

Figure 5 shows the dependences of the angular distributions of atoms of charged amino acid residues of polypeptides A784R98D98 (Fig.5a) and A880R54D54 (Fig.5b) averaged over time intervals with different values of the dipole moment of the nanowire.

It can be seen that, in the case of an unpolarized nanowire, charged amino acid residues Arg and Asp are almost uniformly distributed over the surface, and when the direction of the dipole moment of the nanowire is reversed, the peaks of the angular distributions of the charged units of the polypeptide change in a mirror image.All this means that, in MD simulations at a temperature of $900 \mathrm{~K}$, with a periodic change in the polarization of the nanowire in the transverse direction, the conformational structure of the adsorbed polypeptide on the surface of the metal nanowire periodically changes from a dense enveloping of the nanowire to an elongated conformational structure in the transverse direction to the nanowire twice during one oscillation period. 


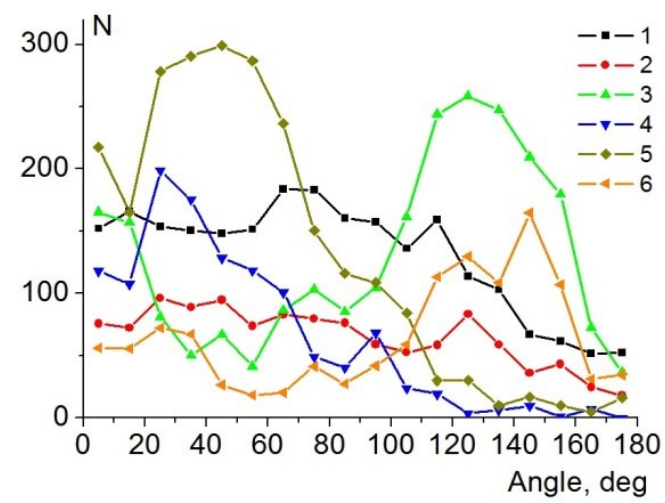

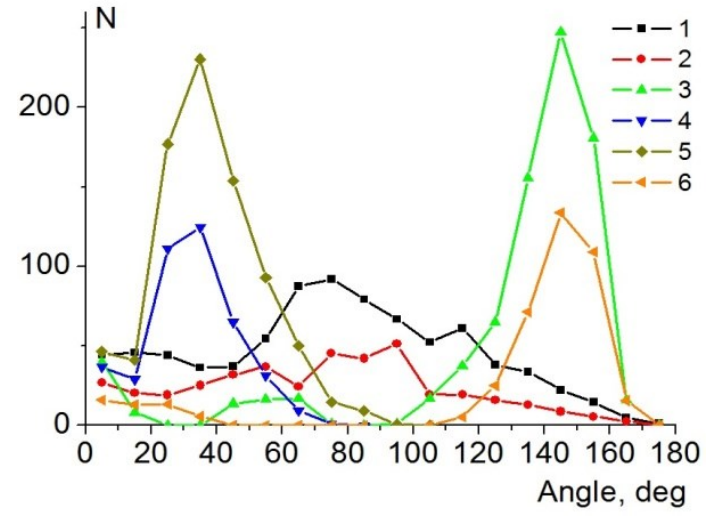

b

Fig.5. Dependences of the average angular distributions of the atoms of the charged $\operatorname{Arg}(1,3,5)$ and Asp $(2,4$, 6) units of the A784R98D98 (a) and A880R54D54 (b) polypeptides over all conformations at the end of the MD simulation at $900 \mathrm{~K}$ on time intervals when nanowire is not polarized $(1,2)$, polarized in the transverse

$$
\text { direction with a dipole moment }+0.97 p_{2 \max }^{n w}(3,4) \text { and }-0.97 p_{2 \max }^{n w}(5,6)
$$

According to the results of MD simulation with a periodic change in time of the polarity in the transverse direction of a gold nanowire with a temperature of $300 \mathrm{~K}$ at the peak value of the dipole moment of the nanowire $p_{1 \max }^{n w}$, a displacement of the links of the adsorbed polyampholyte polypeptide to one side with respect to the plane perpendicular to the direction of the dipole moment of the nanowire and passing through its axis was observed (Fig. 6). The shift took place in the direction (Fig. 6b), where most of the polypeptide units were in the starting conformation (Fig. 1a). In this case, the swelling of the fringe of the adsorbed macrochain occurred, which can be seen if we compare the radial distributions of polypeptide atoms at the end of the MD simulation at $300 \mathrm{~K}$ (Fig. 6e) with the radial density distributions on the surface of the unpolarized nanowire (Figs. 2a and 4a). It can be seen that the peaks of radial distributions for the final conformations of polypeptides obtained at a temperature of $300 \mathrm{~K}$ are located at the surface of the nanowire (Fig. 6e), but they are several times lower than on the surface of an unpolarized nanowire.

The dependences of the angular distributions of the atoms of the adsorbed polypeptides (Figs. $6 \mathrm{c}$ and 6d) show that most of the polypeptide units are on the same side with respect to the horizontal plane passing through the nanowire axis. In this case, the peaks of the angular distributions are in this plane (angle 90 degrees). This region of the nanowire always remains neutral in the process of polarization reversal, and in the adjacent regions from above and below (Figs. 6a and 6b), the surface charges change insignificantly compared to the polar regions (angles of 0 and 180 degrees).

This nature of conformational changes is explained by the fact that at a low temperature of MD simulation, the forces arising as a result of the action of the local electric field are insufficient to overcome the mutual attraction between the polyampholyte links in the weakly charged region of the nanowire. When the nanowire is repolarized in the polar regions along the cross section, strong conformational changes in the macrochain occur, which lead to the fact that the links shift from one pole to the other and, passing through the weakly charged region, are localized in it. In contrast to the case of a spherical nanoparticle [18], where an annular macromolecular fringe was formed, encircling it in the equatorial region, the nanowire has an extended structure. Therefore, a part of the polypeptide units remains in the polar region, which bind amino acid residues, most of which are adsorbed in the weakly charged region (in Figures $6 \mathrm{a}$ and $6 \mathrm{~b}$, on the left and right of the nanowire).

At higher values of the peak dipole moment of the nanowire for the A400R100D100 and A480R60D60 polypeptides at $p_{3 \max }^{n w}$, and for the A784R98D98 and A880R54D54 polypeptides at $p_{2 \max }^{n w}$, desorption of polyampholyte from the nanowire surface took place. In this case, at the first steps of MD simulation, a conformational structure of the macrochainwas formed, similar to the conformations obtained at lower peak values of the dipole moment of the nanowire (Fig. 6). However, then, due to a stronger change in the local electric field, the amplitude of the displacement of charged units in the weakly charged region of the nanowire increased. This led to the fact that the forces of attraction of the macrochain to the nanowire were overcome. 


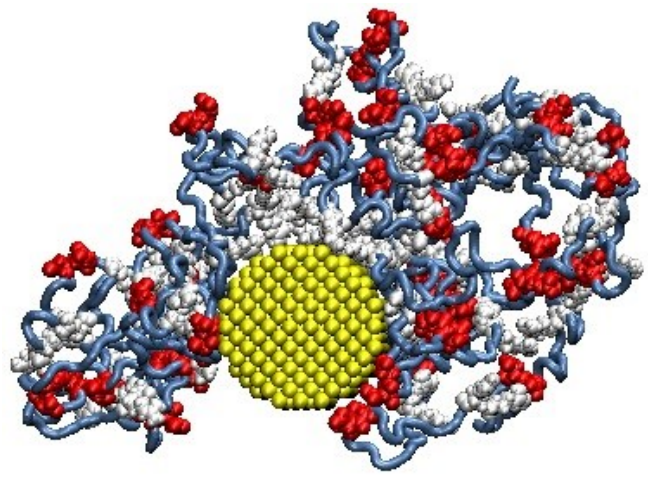

a

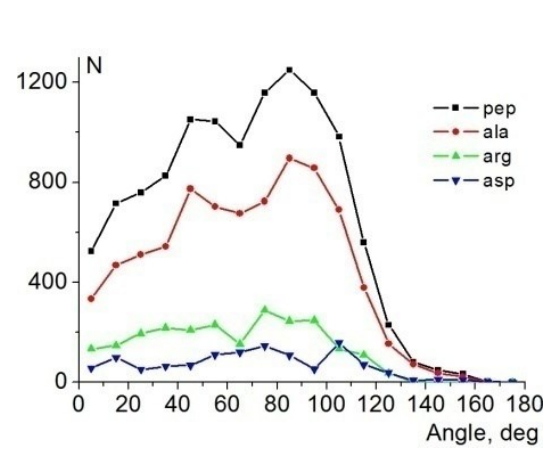

C

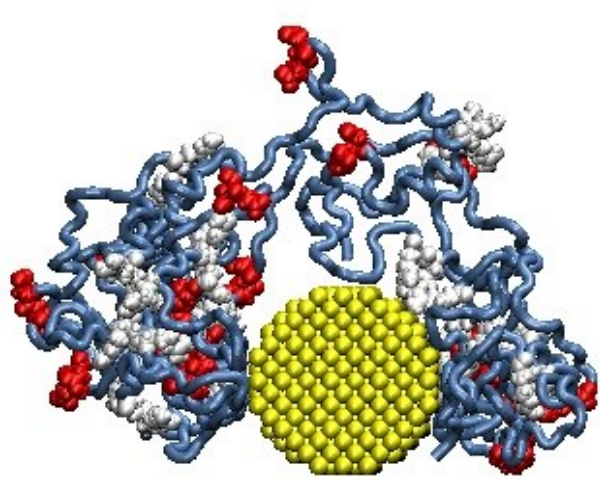

b
Fig.6. Conformations and dependences of the angular distributions of atoms of polypeptides A784R98D98 (a, c) and A880R54D54 (b, d), as well as radial distributions of atomic density (e) of polypeptides A784R98D98 (1) and A880R54D54 (2) after the end of MD simulation with periodic changes polarity of the nanowire in the transverse direction at a temperature of $300 \mathrm{~K}$.

At the same time, due to the generally lower mobility of the links in comparison with the case of the higher temperature of the MD simulation, the movement of charged links between the poles of the nanowire along the cross section did not occur, and the macrochainwas gradually desorbed from the surface.

\section{Analytical model of conformational structure}

Thus, the performed MD simulation showed that the change in the conformations of the macrochains of polyampholyte or polyelectrolyte adsorbed on the nanowire can be carried out by an external electric field polarizing the conductor. In the characteristic case of the transverse orientation of the external homogeneous monochromatic (at frequency $\omega$ ) field intensity vector $\mathbf{E}(t)=\mathbf{E}_{0} \exp (-i \omega t)$ relative to the z-axis of the cylinder, forced oscillations of the surface charge density occur - localized plasmons. In the simplest model, polymer molecules are represented by ideal Gaussian chains, but the attraction of the units to the adsorbent surface is taken into account. Such a model has great clarity, therefore it is convenient for analyzing changes in the conformational structure of the chain with a change in external conditions.

The intensity of the quasi-static field outside a cylinder of radius $\mathrm{R}$, i.e. when $r>R$ and placing it in the field $\mathbf{E}_{0}$ has the form [28]

$$
\mathbf{E}_{2}(r, \varphi)=E_{0} \mathbf{n}_{x}+E_{0} \frac{\varepsilon_{1}(\omega)-\varepsilon_{2}}{\varepsilon_{1}(\omega)+\varepsilon_{2}} \frac{R^{2}}{r^{2}}\left[\left(1-2 \sin ^{2} \varphi\right) \cdot \mathbf{n}_{x}+\sin 2 \varphi \cdot \mathbf{n}_{y}\right]
$$

In the case of a circularly polarized monochromatic electromagnetic field propagating along the $\mathrm{z}$ axis, parallel to the axis of the nanocylinder

$$
\mathbf{E}(t, z)=\left(E_{0} \mathbf{n}_{x}+i E_{0} \mathbf{n}_{y}\right) \exp (i \Omega t-i k z) .
$$

Here, in (2), $\mathbf{n}_{x}, \mathbf{n}_{y}$ are the unit vectors of the coordinate Cartesian axes $\mathrm{x}$ and $\mathrm{y}$. In the long-wavelength limit for the wavenumber $k \rightarrow 0$, the phases $k z, k(z+\Delta z)$ of wave (2) differ little from each other, and 
over the length $l<<1 / k$ we obtain an electric field of strength $E_{0}$ rotating with frequency $\Omega$. In [29], a variant of the circular polarization of the microwave field in the problem of a cylinder with adsorbed macrochainswas considered. In the adiabatically slow version of the field rotation, it is necessary that the frequency $\Omega$ be much lower than the characteristic frequencies of conformational transitions of macrochain fragments $\sim 1 \mathrm{GHz}\left(1 \mathrm{~ns}^{-1}\right)$.

The adsorption potential of the surface of a circular nanowire in the case of van der Waals adsorption can be effectively represented by a combination of the simplest model potentials "solid wall - deltafunctional well": $V_{1}(r)=V_{\infty}(R)-\alpha \delta\left(r-r_{0}\right)$ ( $R$ is the radius of the cylinder) [29]. When a nanowire is placed in a uniform electric field, its potential, as a result of the polarization of the conductor, becomes dependent on the angular variable $\varphi$ in the cross-sectional plane. In an alternating monochromatic microwave field $\mathbf{E}(t)=E_{0}\left(\mathbf{n}_{x}+i \mathbf{n}_{y}\right) \exp (i \Omega t)$, there is a change in polarization according to a harmonic law with frequency $\Omega$, and the potential $-e E_{0} r \cos (\varphi-\Omega t)$ of the external field $\mathbf{E}_{x}(t)$ is added to the adsorption potential $V_{1}(r)$, as well as the potential $V_{\mathrm{P}}$ of the field of the polarized surface of the nanocylinder: $V_{2}(r, \varphi, \Omega t)=-e E_{0} r \cos (\varphi-\Omega t)+V_{\mathrm{P}}(r, \varphi-\Omega t)$. Then the potential of the total field in the space outside the nanowire with the polarized component $\mathbf{E}_{x}(t)$ can be written in the form

$$
V(\mathbf{r}, t)=V_{1}(r)+V_{2}(r, \Phi(t))=V_{\infty}(R)-\alpha \delta\left(r-r_{0}\right)-e E_{0} r \cos \Phi+V_{\mathrm{P}}(r, \Phi),
$$

where phase $\Phi(t)=\varphi-\Omega t$, and

$$
V_{2}(r, \Phi)=-e E_{0} r \cos \Phi+\frac{\varepsilon_{1}(\omega)-\varepsilon_{2}}{\varepsilon_{1}(\omega)+\varepsilon_{2}} R^{2} e E_{0} \frac{\cos \Phi}{r}
$$

- the energy of interaction of the polyelectrolyte link carrying the charge $e$ with the primary external electric field and the polarization field of the wire. The dielectric constants $\varepsilon_{1}(\omega), \varepsilon_{2}$ in (4) characterize the metal of the nanowire and the environment, respectively. The intensity of the quasi-static field $\mathbf{E}_{2}(r, \varphi, t)=-\nabla V_{2} / e$ outside the cylinder, i.e. for $r>R$, when placed in a field $\mathbf{E}(t)$ with circular polarization takes the form

$$
\begin{aligned}
\mathbf{E}_{2}(r, \varphi, t)= & E_{0} \mathbf{n}_{x} \cos \Omega t+E_{0} \mathbf{n}_{x} \frac{\varepsilon_{1}(\omega)-\varepsilon_{2}}{\varepsilon_{1}(\omega)+\varepsilon_{2}} \frac{R^{2}}{r^{2}}\left[\left(1-2 \sin ^{2} \varphi\right) \cdot \cos \Omega t+\sin 2 \varphi \cdot \sin \Omega t\right]+ \\
& +E_{0} \mathbf{n}_{y} \sin \Omega t+E_{0} \mathbf{n}_{y} \frac{\varepsilon_{1}(\omega)-\varepsilon_{2}}{\varepsilon_{1}(\omega)+\varepsilon_{2}} \frac{R^{2}}{r^{2}}\left[\sin 2 \varphi \cdot \cos \Omega t-\left(1-2 \sin ^{2} \varphi\right) \cdot \sin \Omega t\right] .
\end{aligned}
$$

At $\Omega \rightarrow 0$, the circularly polarized field (5) transforms into a static field (1) with linear polarization.

In the case of macrochain polyampholyte, instead of the charge $e$ of the link, one can introduce the effective dipole moment $\mathbf{p}$ of the electrically neutral group of links. Using (5) we can write down the energy $V_{2}^{p}=-\mathbf{p} \mathbf{E}_{2}(r, \varphi, t)$ of interaction of the field $\mathbf{E}_{2}(r, \varphi, \Omega t)$ with the dipole moment $\mathbf{p}$ of the polyampholyte.

In the case of a weak microwave field, taking into account potentials $V_{2}(r, \Phi)$ (4) and $V_{2}^{p}=-\mathbf{p} \mathbf{E}_{2}(r, \varphi, t)$ with field (5) when constructing an analytical model of rearrangement of the conformational structure of a macrochain, as was done, for example, in [17, 29], can be performed within the framework of quasi-static perturbation theory.

Thus, this section presents an analytical model for the rearrangement of the conformational structure of polyampholytic and uniformly charged polypeptides on the surface of a transversely polarized gold nanowire. If necessary, the results obtained can be easily generalized, including for the case of rotation of the polarizing electric field vector.To solve a differential equation of Schrödinger type [30]

$$
\frac{a^{2} k T}{6} \nabla^{2} \psi(\mathbf{r})=[V(\mathbf{r})-\varepsilon] \psi(\mathbf{r})
$$


where $a$ is the size of the chain link that determines the conformational function $\psi(\mathbf{r})$; in the case of a weak microwave field, the potential (3) can be taken into account within the framework of perturbation theory. Equation (6) with potential (3) contains an angular variable, so it should be written in the form

$$
\frac{a^{2} k T}{6}\left[\frac{1}{r} \frac{\partial}{\partial r} r \frac{\partial}{\partial r}+\frac{1}{r^{2}} \frac{\partial^{2}}{\partial \varphi^{2}}\right] \psi(\mathbf{r})=[V(\mathbf{r})-\varepsilon] \psi(\mathbf{r}) \text {. }
$$

Solution (7) can be represented as $\psi(\mathbf{r})=F(r) \Phi_{m}(\varphi)$, where $\Phi_{m}(\varphi)$ is the eigenfunction of the projection operator of the quantum orbital angular momentum with integer $m$ : $\Phi_{m}(\varphi)=1 / \sqrt{2 \pi} \exp (\operatorname{im} \varphi)$.

Then, taking into account the spectrum of eigenvalues for the functions $\Phi_{m}(\varphi)$

$$
\frac{\partial^{2} \Phi_{m}}{\partial \varphi^{2}}=-m^{2} \Phi_{m}(\varphi)
$$

for the radial function $F_{l}(r)$ we obtain the equation

$$
\frac{a^{2} k T}{6}\left[\frac{1}{r} \frac{d}{d r} r \frac{d}{d r}-\frac{m^{2}}{r^{2}}\right] F_{m}(r)=\left[V(r)-\varepsilon_{m}\right] F_{m}(r) .
$$

Here in (9) the radial potential $V(r)=V_{1}(r)+V_{2}(r)$, and $V_{2}(r)$ is defined by the relation $V_{2}(r, \theta)=V_{2}(r) \Phi_{1}(\varphi)$, or

$$
\begin{aligned}
& V_{2}(r, \varphi)=-\left[1-\alpha^{\prime}(\omega)\left(\frac{R}{r}\right)^{2}\right] r E_{0} \cos \varphi \\
& V_{2}(r, \varphi)=-e E_{0} r \cos \varphi+\frac{\varepsilon_{1}(\omega)-\varepsilon_{2}}{\varepsilon_{1}(\omega)+\varepsilon_{2}} R^{2} e E_{0} \frac{\cos \varphi}{r}
\end{aligned}
$$

For ease of writing (4), it is assumed that the frequency $\Omega \rightarrow 0$ (quasi-static mode). For the function $F_{m}(r)$, we obtain the equation

$$
F_{m}^{\prime \prime}(r)+\frac{1}{r} F_{m}^{\prime}(r)-\frac{m^{2}}{r^{2}} F_{m}(r)=\frac{6}{a^{2} k T}\left[V_{1}(r)-\varepsilon_{l}\right] F_{m}(r),
$$

which in the absence of potential $V_{1}(r)=V_{\infty}(R)-\alpha \delta\left(r-r_{0}\right)$ is the Bessel equation for cylindrical functions $Z_{m}(\xi)$. Thus, the solution to (10) is the Bessel functions of the imaginary argument $I_{m}\left(q_{m} r\right)$ and $K_{m}\left(q_{m} r\right), \xi=q_{m} r, q_{m}^{2}=-\frac{6 \varepsilon_{m}}{a^{2} k T}$. As solutions to equation (6) with a certain index $m$ in the field $V(r)=V_{1}(r)$, decaying at infinity, we can write the following

$$
\left\{\begin{array}{ll}
F_{m}^{I}(r)=A_{m} I_{m}\left(q_{m} r\right)+B_{m} K_{m}\left(q_{m} r\right), & R<r<r_{0} \\
F_{m}^{I I}(r)=C_{m} K_{m}\left(q_{m} r\right), & r_{0}<r<\infty
\end{array} .\right.
$$

Constants $A_{m}, B_{m}$ and $C_{m}$ we find from the considerations that $\psi$ - functions must satisfy the following boundary conditions

$$
\psi_{I}(R)=0, \quad \psi_{I}\left(r_{0}\right)=\psi_{I I}\left(r_{0}\right), \quad \psi_{I I}^{\prime}\left(r_{0}\right)-\psi_{I}^{\prime}\left(r_{0}\right)=-\frac{6 \alpha}{a^{2} k T} \psi_{I I}\left(r_{0}\right) .
$$

The last equation allows you to determine a single discrete spectrum level $\varepsilon_{m}$ for each $m$. For $m=0$, we arrive at the problem with circular symmetry, which we have already investigated in a number of works.In the general case of an arbitrary index $m$ to determine the parameters $q_{m}$ and $\varepsilon_{m}$ (the important 
case $m=1$ ), it is necessary to use the equation

$$
\left.\left.\frac{d}{d r} F_{m}^{I I}(r)\right|_{r=}-{ }_{u \prime} \quad=-{ }_{u n} \quad r_{0}\right) \text {. }
$$

From (12) we find the relations between the constants $A_{m}, B_{m}$ and $C_{m}$ :

$$
B_{m}=-A_{m} \frac{I_{m}\left(q_{m} R\right)}{K_{m}\left(q_{m} R\right)}, \quad C_{m}=A_{m}\left[\frac{I_{m}\left(q_{m} r_{0}\right)}{K_{m}\left(q_{m} r_{0}\right)}-\frac{I_{m}\left(q_{m} R\right)}{K_{m}\left(q_{m} R\right)}\right] .
$$

Then solutions (11) take the form

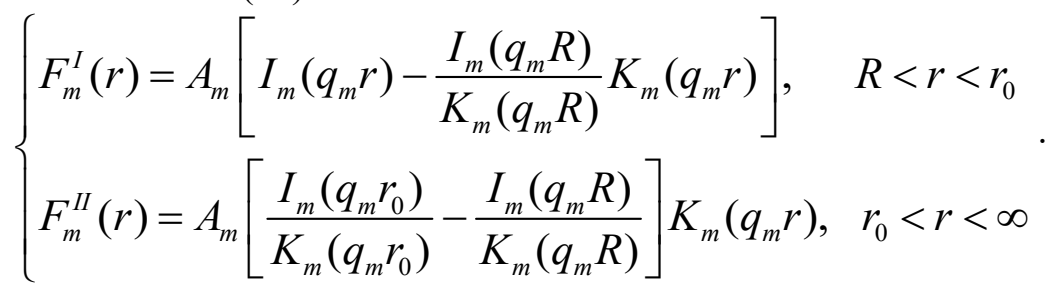

Substituting (11') into (13) we obtain the general transcendental equation for the eigenvalues $q_{m}$

$$
\frac{a^{2} k T}{6 \alpha r_{0}}=\quad-\quad \quad \mathbf{1}_{m}\left(y_{m} R\right) .
$$

For $m=1$, from (11) we obtain

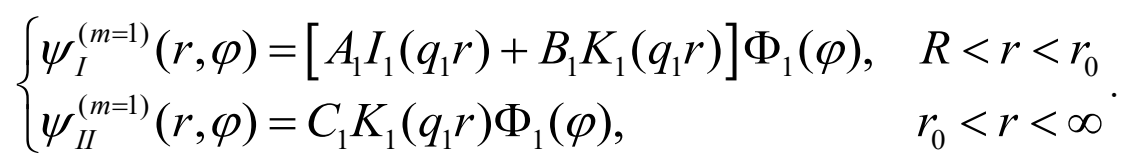

Note that the density $n(r, \varphi)=\psi^{2}(r, \varphi)$ of the units of the adsorbed macrochain will still be determined by functions (11) or their equivalent (11') with the index $m=0$, but not (16), since the eigenvalues $\varepsilon_{1}$ corresponding to (16) have a larger modulus than $\varepsilon_{0}$. In addition, let us pay attention to the fact that the first-order corrections to the eigenvalue $\varepsilon_{0}$, taking into account the disturbance $V_{2}(r, \varphi)=-e E_{0} r \cos \varphi+V_{P}(r, \varphi)$, are equal to zero for states (11) and (11'). Correct use of the Rayleigh-Schrödinger perturbation theory requires taking into account states (16) to refine the basic state $F_{0}(r)$.

\section{Microwave perturbation theory}

Taking into account the orthogonality $\Phi_{m}(\varphi)$ with different indices $m$ and the structure of the angular dependence of the perturbation operator $V_{2}(r, \varphi) \sim \cos \varphi$, we note that only functions (16) with $m=1$ will contribute to the basic state corrections $\psi_{I, I I}^{(m=0)}(r)$. The corrected basic state $\psi_{I, I I}^{(m=0)}(r)$ in the second order in the small parameter $\eta=e E_{0} a /\left|\varepsilon_{1}-\varepsilon_{0}\right|$ can be written as

$$
\begin{cases}\psi_{I}(r, \varphi)=\psi_{I}^{(m=0)}(r)+\frac{\left\langle\psi_{I}^{(m=0)}\left|V_{2}\right| \psi_{I}^{(m=1)}\right\rangle}{\varepsilon_{1}^{(0)}-\varepsilon_{0}^{(0)}} \psi_{I}^{(m=1)}(r, \varphi)-\frac{1}{2} C_{I}^{(2)} \psi_{I}^{(m=0)}(r), & R<r<r_{0} \\ \psi_{I I}(r, \varphi)=\psi_{I I}^{(m=0)}(r)+\frac{\left\langle\psi_{I I}^{(m=0)}\left|V_{2}\right| \psi_{I I}^{(m=1)}\right\rangle}{\varepsilon_{1}^{(0)}-\varepsilon_{0}^{(0)}} \psi_{I I}^{(m=1)}(r, \varphi)-\frac{1}{2} C_{I I}^{(2)} \psi_{I I}^{(m=0)}(r), & r_{0}<r<\infty\end{cases}
$$

Expressions for the radial-angular distribution of the density of the units of the adsorbed Gaussian macrochain can be written in the form

$$
\begin{array}{ll}
n_{I}(r, \varphi)=n_{I}^{(m=0)}(r)+n_{I}^{(1)}(r) \cos \varphi+n_{I}^{(2)}(r) \cos ^{2} \varphi, & R<r<r_{0}, \\
n_{I I}(r, \varphi)=n_{I I}^{(m=0)}(r)+n_{I I}^{(1)}(r) \cos \varphi+n_{I I}^{(2)}(r) \cos ^{2} \varphi, & r_{0}<r<\infty,
\end{array}
$$


where

$$
n_{I, I I}^{(1)}(r)=2 \frac{\left\langle\psi_{I, I I}^{(m=0)}\left|V_{2}\right| \psi_{I, I I}^{(m=1)}\right\rangle}{\varepsilon_{1}^{(0)}-\varepsilon_{0}^{(0)}} F_{0}^{I, I I}(r) F_{1}^{I, I I}(r), \quad n_{I, I I}^{(2)}(r)=\frac{\left|\left\langle\psi_{I}^{(m=0)}\left|V_{2}\right| \psi_{I}^{(m=1)}\right\rangle\right|^{2}}{\left|\varepsilon_{1}^{(0)}-\varepsilon_{0}^{(0)}\right|^{2}}\left|F_{1}^{I, I I}(r)\right|^{2} .
$$

We find the eigenvalues $\varepsilon_{1}$ from the equation $\varepsilon_{l}=-q_{l}^{2} a^{2} k T / 6$, and the coefficients $\left\langle\psi_{I, I I}^{(m=0)}\left|V_{2}\right| \psi_{I, I I}^{(m=1)}\right\rangle$ are the following integrals

$$
\left\langle\psi_{I, I I}^{(m=0)}\left|V_{2}\right| \psi_{I, I I}^{(m=1)}\right\rangle=\left\{\begin{array}{ll}
\int_{R}^{r_{0}} F_{0}^{I}(r) V_{2}(r) F_{1}^{I}(r) r d r, & I: R<r<r_{0} \\
\int_{r_{0}}^{\infty} F_{0}^{I I}(r) V_{2}(r) F_{1}^{I I}(r) r d r, & I I: r_{0}<r<\infty
\end{array},\right.
$$

where radial functions $F_{m=0 ; 1}^{I, I I}(r)$ are defined by expressions (11'). Calculations of the radial-angular distributions of the density of the links of the macrochain can be made on the basis of expressions (18-19).

\section{Conclusion}

Thus, as a result of MD simulation of polyampholyte polypeptides on the surface of a metal nanowire with periodic changes in time of its polarity in the transverse direction with an ultrahigh frequency, significant conformational changes of the adsorbed macromolecule are observed. In the case of MD simulation with a temperature high enough to overcome theinterlink potential barriers of the macrochain adsorbed on the nanowire surface, temporary fluctuations in the polyampholyte conformations were observed. The conformational structure of the adsorbed polyampholyte polypeptide for half the period of change in the dipole moment of the nanowire changed from dense enveloping of the nanowire to the conformation in which the macromolecular fringe was extended in the transverse direction along the dipole moment of the nanowire, at those times when the dipole moment of the nanowire had the maximum absolute values.

At a comparatively low temperature of MD simulation and sufficiently low values of the nanowire dipole moment, a gradual swelling of the fringe of the adsorbed polyampholyte was observed with a displacement of most of its links to one side with respect to the plane perpendicular to the direction of the nanowire dipole moment and passing through its axis. In this case, the peaks of the angular distributions of the atoms of the adsorbed polypeptides were in this plane. At higher values of the dipole moment at a given temperature of MD simulation, such swelling of the macromolecular fringe led to desorption of polyampholyte from the nanowire surface.

Such changes in the conformational structure of polyampholyte adsorbed on the surface of the nanowire, induced by microwave electromagnetic radiation, will have a significant effect on the photochemical processes occurring in the layer of the macromolecular fringe formed by this polypeptide.

Therefore, they can be used to create and modify sensors with a conformational structure of macromolecules tunable under the influence of electromagnetic radiation, such as sensors based on the effects of surface plasmon resonance and surface-enhanced Raman scattering, as well as luminescenceoptical meters for the concentration of molecular oxygen in the ground or electronically excited state.

In a number of works, including the work of the authors of [20], it was noted that plasmonic nanoparticles and nanowires significantly change the rate of spontaneous radiative transitions in electronically excited molecules located near conducting nanobodies. This circumstance allows one to consider plasmonnanoantennas as control elements in molecular luminescent sensors of chemical compounds. The mechanism for increasing the intensity of the spontaneous glow of the sensitive elements of such sensors is associated with the plasmon resonance in the nanoantenna, which is determined by the geometric parameters of the latter. In spherical nanoparticles, the oscillations of surface plasmonshave been studied in sufficient detail, in contrast to extended quasi-one-dimensional conducting lines. Surface plasmon resonances in a cylinder can be determined by both local transverse oscillations of the electron density and waves traveling along the axis with different mode structures. Only further studies will make it possible to 
determine which of the plasmon resonances in cylindrical bodies and shells will be more important for the formation of luminescent signals in composite, molecular-plasmonic nanostructures with axial symmetry.

\section{Acknowledgments}

This work was supported by the Ministry of Science and Higher Education of the Russian Federation within the framework of project No. FSGU-2020-0003.

\section{REFERENCES}

1 Li D., MaYa., Duan H., et al. Griess reaction-based paper strip for colorimetric/fluorescent/SERS triple sensing of nitrite. BiosensBioelectron. 2018, Vol. 99, pp. $389-398$.

2 Feng J., Xu L., Cui G., et al. Building SERS-active hetero assemblies for ultrasensitive Bisphenol A detection. Biosens Bioelectron. 2016, Vol. 81, pp. 138-142.

3 Zhao X., Dong J., Cao E., et al. Plasmon-exciton coupling by hybrids between graphene and gold nanorods vertical array for sensor. Applied Materials Today. 2019, Vol. 14, pp. 166-174.

4 Yilmaz M., Senlik E., Biskin E., et al. Combining 3-D plasmonic gold nanorod arrays with colloidal nanoparticles as a versatile concept for reliable, sensitive, and selective molecular detection by SERS. Phys. Chem. Chem. Phys. 2014, Vol. 16, p. 5563-5570.

5 Chandran G.T., Jha G., Qiao S., et al. Supercharging a MnO2 nanowire: an amine-altered morphology retains capacity at high rates and mass loadings.Langmuir,2017, Vol. 33, pp. 9324-9332.

6 Rong Y., Song L., Si P., et al. Macroscopic assembly of gold nanorods into superstructures with controllable orientations by anisotropic affinity interaction.Langmuir,2017, Vol. 33, pp. 13867-13873.

7 Azman N.A., Thanh N.X., Kah J.C.Y. Sequestration of Cetyltrimethylammonium Bromide on Gold Nanorods by Human Serum Albumin Causes Its Conformation Change. Langmuir, 2020, Vol. 36, pp. 388-396.

8 Kesal D., Christau S., Krause P., et al.Uptake of pH-Sensitive Gold Nanoparticles in Strong Polyelectrolyte Brushes. Polymers, 2016,Vol. 8, p. 134.

9 Lee J., Chung K., Lee J., et al. LSPR Coupling: In Situ Studies of Surface-Plasmon-Resonance-Coupling Sensor Mediated by Stimuli-Sensitive Polymer Linker. Adv. Funct. Mater.2015, Vol. 25, pp. 6716-6724.

10 Chen Y., Cruz-Chu E.R., Woodard J., et al. Electrically Induced Conformational Change of Peptides on Metallic Nanosurfaces. ACS Nano, 2012, Vol. 6, pp. 8847-8856.

11 Sotnikov D.V., Zherdev A.V., Dzantiev B.B. Detection of Intermolecular Interactions Based on Surface Plasmon Resonance Registration. Biochemistry (Moscow), 2015, Vol. 80, pp. 1820-1832.

12 Zengin A., Tamer U., CaykaraT..A new plasmonic device made of gold nanoparticles and temperature responsive polymer brush on a silicon substrate. J Colloid Interface Sci.2015, Vol. 448, pp. 215-221.

13 Chen H., You T., Xu G., et al. Humidity-responsive nanocomposite of gold nanoparticles and polyacrylamide brushes grafted on Ag film: synthesis and application as plasmonic nanosensor. Sci. China Mater. 2018, Vol. 61, pp. 1201-1208.

14 Kruchinin N.Yu., Kucherenko M.G.Molecular dynamics simulation of electrically induced conformational changes of polyampholytic polypeptides on gold nanoparticle surface. Colloid Journal, 2019, Vol. 81, pp. 110-119.

15 Kruchinin N.Yu., Kucherenko M.G. Electrically induced conformational changes in gold cluster-bonded polyampholytic polypeptides on a surface of gold: molecular dynamic simulation. Russian Journal of Physical Chemistry A, 2020, Vol. 94, pp. 1433-1438.

16 Kruchinin N.Yu., Kucherenko M.G.A Molecular dynamics simulation of polyampholytic polypeptides associated with atomic clusters on the surfaces of metal-like nanoobjects. Biophysics, 2020,Vol. 65, pp. 186-194.

17 Kruchinin N.Yu., Kucherenko M.G. Molecular-dynamics simulation of rearrangements in the conformational structure of polyampholytic macromolecules on the surface of a polarized metal nanoparticle. Colloid Journal, 2020, Vol. 82, pp. 136-143.

18 Kruchinin N.Yu., Kucherenko M.G. Conformational rearrangements of polyampholytic polypeptides on metal nanoparticle surface in microwave electric field: molecular-dynamics simulation. Colloid Journal, 2020, Vol. 82, pp.392-402.

19 Kucherenko M.G., Stepanov V. N., Kruchinin N. Yu. Inter molecular non radiative energy transfer in clusters with plasmonic nanoparticles. Optics and Spectroscopy, 2015, Vol. 118, pp. 103-110.

20 Kucherenko M.G., Stepanov V. N., Kruchinin N. Yu. Plasmon activation and luminescence quenching of solutions of polyphenylenevinylene (MEH-PPV) by single-walled and double-walled carbon nanotubes. Optics and Spectroscopy, 2020, Vol. 128, pp. 1298-1310.

21 Kucherenko M.G., Rusinov A.P., Chmereva T.M., et al.Kinetics of photoreactions in a regular porous nanostructure with cylindrical cells filled with activator-containing macromolecules. Optics and Spectroscopy, 2009, Vol. 107, pp. 480-485. 
22 Kucherenko M. G., Izmodenova S.V., KruchininN.Yu., Chmereva T.M. Change in the kinetics of delayed annihilation fluorescence during rearrangement of polymer-chain structure in a nanocavity of a solid adsorbent. High Energy Chemistry, 2009, Vol. 43, pp. 592 - 598.

23 Phillips J.C., Braun R., Wang W., et al. Scalable molecular dynamics with NAMD. J Comput Chem. 2005, Vol. 26, pp. 1781-1802.

24 MacKerell A.D. Jr., Bashford D., Bellott M., et al. All-atom empirical potential for molecular modeling and dynamics studies of proteins J. Phys. Chem. B. 1998, Vol. 102, pp. 3586-3616.

25 Heinz H., Vaia R.A., Farmer B.L., Naik R.R. Accurate Simulation of Surfaces and Interfaces of FaceCentered Cubic Metals Using 12-6 and 9-6 Lennard-Jones Potentials .J. Phys. Chem. C.2008, Vol. 112, pp. 1728117290 .

26 Darden T., York D., Pedersen L. Particle mesh Ewald: An N·log(N) method for Ewald sums in large systems. J.Chem. Phys. 1993, Vol. 98, pp. 10089-10092.

27 Jorgensen W.L., Chandrasekhar J., Madura J.D., et al. Comparison of simple potential functions for simulating liquid water.J. Chem. Phys. 1983, Vol. 79, pp. 926-935.

28 Novotny L., Hecht B. Principles of nanooptics. Cambridge: Cambridge University Press. 2006, 564p.

29 Kruchinin N.Yu., Kucherenko M.G. Rearrangements in the conformational structure of polypeptides on the surface of a metal nanowire in rotating electric field: molecular dynamics simulation. Colloid Journal, 2021, Vol. 83, pp. $79-87$.

30 Grosberg A.Y., Khokhlov A.R., Pande V.S. Statistical Physics of Macromolecules. New York: AIP Press. 1994. $347 \mathrm{p}$. 